\title{
TTR
}

Traduction, terminologie, re?daction

\section{Traduire Charles S. Peirce. Le signe: le concept et son usage}

\section{Gérard Deledalle}

Volume 3, numéro 1, 1er semestre 1990

L'agora de la traduction

URI : https://id.erudit.org/iderudit/037056ar

DOI : https://doi.org/10.7202/037056ar

Aller au sommaire du numéro

Éditeur(s)

Association canadienne de traductologie

ISSN

0835-8443 (imprimé)

1708-2188 (numérique)

Découvrir la revue

Citer cet article

Deledalle, G. (1990). Traduire Charles S. Peirce. Le signe : le concept et son

usage. TTR, 3(1), 15-29. https://doi.org/10.7202/037056ar d'utilisation que vous pouvez consulter en ligne.

https://apropos.erudit.org/fr/usagers/politique-dutilisation/ 


\section{Traduire Charles S. Peirce Le signe: le concept et son usage}

\section{Gérard Deledalle}

Nous sommes tous des traducteurs: auteurs, traducteurs ou lecteurs, que nous écrivions ou lisions dans notre langue maternelle ou dans une langue apprise. L'auteur traduit sa pensée pour la faire comprendre à un public donné; le traducteur, en quelque langue que ce soit, pour son public à un moment donné de l'histoire; le lecteur pour comprendre ce qu'il lit en le traduisant, c'est-à-dire en le transposant dans son propre monde mental, qu'il ait dix ans ou cinquante ans, qu'il sache à peine lire ou qu'il soit un spécialiste de la question dont traite le livre qu'il est en train de lire et qu'il n'a pas écrit lui-même. On voit à quel point est vraie et combien doit être nuancée l'expression italienne: traduttore traditore. Toute écriture et toute lecture étant traduction, toute écriture et toute lecture est trahison.

Pour comprendre, il ne suffit pas de traquer la trahison pour retrouver le vrai, car il n'y a pas une écriture, une lecture qui serait vraie en soi. Pour comprendre, il faut expliquer pourquoi il paraît $\mathrm{y}$ avoir trahison, autrement dit, traduire la trahison de manière à la justifier.

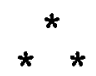

Le cas de Charles S. Peirce est exemplaire à cet égard, en tant qu'auteur d'abord: pourquoi change-t-il si souvent de terminologie, lui qui s'est institué théoricien de l'éthique terminologique, et gardien vigilant, sa vie durant, de la morale terminologique? Le 
commentateur, qu'il soit de langue anglaise ou autre, confronté au texte anglais - et tout lecteur de langue anglaise en fait - doit-il prendre les mots au pied de la lettre ou, au contraire, se mettre à la place de l'auteur, autant que faire se peut, et essayer de découvrir à qui l'auteur s'adresse, pour comprendre le pourquoi de la terminologie employée? Le traducteur de Peirce enfin, qu'il soit allemand, français ou italien, doit-il s'en tenir aux mots qu'il lit dans le texte original ou doit-il traduire la pensée de l'auteur en tenant compte du public de l'auteur ou de son propre public?

Voilà une foule de questions auxquelles $\mathrm{j}$ 'aimerais, en tant que traducteur et commentateur de Peirce en français, répondre. Il n'y a pas, en fait, autant de questions qu'il n'y paraît et il ne sera pas très difficile d'y voir clair si l'on examine des cas précis.

Commençons par la dernière question posée: le traducteur doit-il traduire la pensée de l'auteur dans la terminologie du lecteur? La réponse est claire et nette. Elle est négative. Je ne soutiendrai pas que je n'ai jamais moi-même péché. Il m'est arrivé pour faire comprendre Peirce, de dire en commentaire, non en traduction: "Cela correspond à tel concept chez tel auteur de langue française». Prenons le concept de signe, par exemple. Le signe peircien est triadique. Il comprend trois termes: un representamen (Representamen), un objet (Object) et un interprétant (Interpretant), dont Peirce dit de chacun d'eux qu'il est un signe, tout en insistant sur le caractère indécomposable de la triade sémiotique. Le signe saussurien est dyadique. Il est le couple également indissoluble formé d'un signifiant et d'un signifié. Faut-il traduire Representamen (le representamen) par «signifiant» pour que le lecteur français comprenne ce qu'est le premier terme de la triade peircienne? Lequel des deux termes restants est le signifié? Object (l'objet) ou Interpretant (l'interprétant)? Bien malin celui qui le dira. Certains n'ont pas hésité cependant à traduire Interpretant par «signifié» et Object par «référent». Non sans quelque justification apparente. Peirce ne dit-il pas que l'interprétant est «le résultat signifié d'un signe» (significate outcome of a sign) (5.473, ES:128) ${ }^{1}$. Et certains n'ont-ils pas trouvé dans Saussure un «référent» qui en ferait une théorie triadique?

1. Les références dans le texte renvoient aux Collected Papers de Peirce: Cambridge, Mass., Harvard University Press, vol. 1-6, Charles Hartshorne et Paul Weiss, éd., 1931-1935; vol. 7-8, Arthur W. Burks, éd., 1958. Le premier chiffre renvoie au volume, les autres au paragraphe dans le volume. «ES» désigne notre traduction des Écrits sur le signe de Peirce (Paris, Éditions du Seuil, 1978) et les chiffres qui suivent renvoient à la page dans ce volume. 
Même si l'on continuait ce petit jeu de trahison, on se heurterait à d'autres problèmes. Ainsi le mot «signe» (définition mise à part) peut-il être employé indifféremment en français avec une signification peircienne et saussurienne? (Je dis «signification» et non «sens», car le «sens» chez Peirce - et Dewey - est définitionnel.) La réponse, ici encore, est négative. Le mot «signe» chez Peirce est une abréviation pour «signe-action», opposé à «signerepresentamen». Si l'on voulait être tout à fait exact, il faudrait tantôt, lorsque l'on parle formellement du signe, dire qu'il est un representamen, - ce que sont le representamen lui-même, l'objet et l'interprétant définis formellement comme éléments du signe triadique, - tantôt lorsque l'on parle du signe-action, c'est-à-dire du processus par lequel un signe-interprétant dit que tel signe-objet est l'objet de ce signe-representamen - ce processus, Peirce l'appelle semiosis que je traduis par «sémiose» quand je n'utilise pas le décalque «semiosis». Le signe saussurien est toujours formel, mais il peut acquérir des valeurs différentes en des lieux et à des moments différents. Dira-t-on que la "sémiose» est l'équivalent de la «valeur»? Ne serait-ce pas plutôt l'interprétant? Arrêtons-nous, car il arrive un temps où le traître ne s'y retrouve plus et se trahit lui-même.

Revenons en arrière. Admettons l'entêtement de fait des auteurs, traducteurs et lecteurs, et parlons de signe indifféremment chez Peirce et chez Saussure. Quand un signe cesse-t-il d'être un signe chez Peirce et chez Saussure? Et l'on trouve immédiatement une nouvelle raison d'être prudent quand on traduit sign par «signe», car le signe saussurien est linguistique. Le signe peircien peut l'être aussi certes, mais il n'est pas linguistique en tant que tel. Il est soit un qualisigne, soit un sinsigne, soit un légisigne c'est en tant que légisigne qu'il peut être linguistique: le signe linguistique est donc pour Peirce un type de légisignes parmi d'autres. Encore faut-il préciser s'il est symbolique, indiciaire ou iconique.

Arrêtons-nous un instant ici sur chacun de ces termes. «Iconique», adjectif de «icône» semble aussi vieux, sinon que le monde, du moins que la peinture figurative et plus particulièrement, que la peinture byzantine. Or il n'en est rien. Si le mot s'emploie en anglais depuis le XVIII' siècle, et s'il a bien été introduit en théorie des signes par l'intermédiaire de l'anglais, c'est à l'usage qu'en fait Peitce qu'on se réfère toujours, mais en un sens réducteur. Est iconique chez Peirce un signe ou plus exactement un representamen qui renvoie à un objet qui lui ressemble: un representamen iconique peut donc renvoyer à luimême. Or, on limite en général le sens d'iconique à la ressemblance à quelque chose d'autre que le signe. Ce que peut également 
faire le representamen iconique chez Peirce, mais la différence est d'importance. Voici la définition de Peirce:

Une icône est un signe qui renvoie à l'objet qu'il dénote simplement en vertu des caractères qu'il possède, que cet objet existe réellement ou non. (2.247, ES: 140)

Quant à l'adjectif «indiciaire», du substantif «indice», il est fort souvent - trop souvent à mon goût - traduit par «indexical» Je reconnais que ce que je traduis par «indice» et «indiciaire» est ce que Peirce appelle index et indexical. Cependant, outre que «indexical» n'est pas français, lui donner pour racine «index» est un contresens, bien que Peirce donne pour exemple parfait d'indice l'index de la main que l'on pointe vers un objet. Ma cause semblerait perdue si l'on s'en tenait à cet exemple. Or, ce que Peirce nomme index (pluriel: indices) est «un signe qui renvoie à l'objet qu'il dénote parce qu'il est réellement affecté par cet objet» (2.248, ES: 140). Et Peirce de donner de nombreux exemples de ce que le français appelle couramment «indice», que ce soit un baromètre bas et un air humide qui est indice de pluie, une girouette qui indique la direction du vent et aussi, bien sûr, l'étoile polaire et "l'index que l'on pointe pour montrer dans quelle direction se trouve le nord» (2.286, ES: 154). Et beaucoup d'autres encore, authentiques comme ces derniers: tous les mots que les linguistes appellent aujourd'hui «déterminants»: tous les pronoms et les prépositions et bien d'autres expressions comme «le premier», «le dernier», «à droite», «à gauche», etc. (2.289-290, ES: 157-158), et même le nom propre; et aussi des indices, certes dégénérés (parce qu'ils renvoient aux signes des choses et non aux choses ellesmêmes) comme les lettres en géométrie et en algèbre et les indices en mathématiques en général (2.305, ES: 160).

Le traducteur du livre d'Umberto Eco, Segno, voulait traduire en français les deux termes qu'emploie Eco pour traduire index en italien, à savoir: indice et indizio, par le seul terme français «indice». Umberto Eco s'y opposa en disant qu'il fallait distinguer l'index motivé (traduit en italien par indice) de l'index immotivé (traduit en italien par indizio). Or, ce faisant, on introduit une distinction saussurienne dans un contexte peircien qui ne l'admet pas. On trouve donc dans le Signe qui est le titre du livre d'Eco en français: «Index» («indexical») pour la tache d'eau qui est «un indizio de la pluie, alors que la flèche $(\rightarrow)$ est un indice», un «indice» («indiciaire») 2 .

2. Umberto Eco, le Signe, trad. Jean-Marie Klinkenberg, Collection «Média», dirigée par Jacques Dubois (Bruxelles, Éditions Labor, 1988), p. 62, note 1. 
Y a-t-il vraiment une raison à cette distinction? Ma réponse est ici encore négative. La triadicité peircienne implique par définition une troisième dimension qui est celle du «symbole». Selon Peirce, la flèche $(\rightarrow)$ est un symbole qui comprend, suivant la hiérarchie des catégories dont nous reparlerons, à plusieurs reprises, un indice et une icône. Le symbole, écrit Peirce,

est un signe qui renvoie à l'objet qu'il dénote en vertu d'une loi, d'ordinaire une association d'idées générales, qui détermine l'interprétation du symbole par référence à cet objet. (2.249, ES: 140-141)

Pour Peirce, le symbole est, à strictement parler, un légisigne symbolique, ce qui ne veut pas dire que tout légisigne soit symbolique. Le signe linguistique, par exemple, est un légisigne qui peut, suivant les cas, être symbolique, indiciaire ou iconique. La distinction que propose Eco entre «index» et «indice», pour nécessaire qu'elle soit pour un dualiste d'obédience saussurienne, ne l'est pas pour un Peircien pour qui les catégories de pensée sont ordinales et non cardinales.

Un mot donc sur cette nouvelle distinction catégorielle. La sémiotique de Peirce repose sur une conception ordinale des catégories. L'homme dans l'univers où il vit est confronté à trois dimensions: au sentiment (ou senti) lié à la qualité, à l'existant (ou fait individuel concret) et à la pensée (ou règle à laquelle se plie aussi bien la loi, en quelque sens que ce soit, que le discours). Il y a donc trois dimensions: 1,2 et 3 . Ce sont intellectuellement parlant des catégories. Conçues en termes cardinaux, ces trois dimensions ou catégories sont hétérogènes, sans rapport l'une avec l'autre; conçues en termes ordinaux, elles sont hiérarchisées: le 3 est troisième et présuppose le 2 et le 1 ; le 2 est second et présuppose le 1; le 1 est premier et ne présuppose rien en dehors de lui-même. En conséquence, la règle présuppose l'existant qui présuppose sa propre possibilité d'exister.

Toute la sémiotique phanéroscopique de Peirce repose sur cette hiérarchie: l'ordre est troisième: loi des existants; l'existant est ce qu'il est, il est second: il obéit ou n'obéit pas au troisième, mais ne peut lui donner d'ordre et il ne peut être qu'à la condition d'avoir été possible; le possible est premier: il ne peut ni s'autoordonner à l'exister second, et moins encore donner d'ordre à la loi troisième.

Il faut avoir tout cela à l'esprit pour traduire et comprendre Peirce. Mais comment y parvenir? 
Lire Peirce et donc le traduire exige essentiellement qu'on replace le texte de Peirce dans son contexte. Il faut, pour ce faire, répondre à deux questions: 1) À quel moment de l'histoire de la pensée de Peirce ce texte a-t-il été écrit? 2) A qui s'adressait-il? Ce n'est qu'en possession des réponses à ces questions que le lecteur anglophone pourra comprendre et le traducteur trouver dans sa propre langue le mot ou l'expression ou la tournure qui convient pour faire comprendre le texte de Peirce.

I. Prenons un premier exemple pour répondre à la première question. Il se rapporte aux catégories dont nous venons de parler. Peirce écrit en 1867 dans un article intitulé «Sur une nouvelle liste de catégories», les deux textes suivants que je commenterai.

Les cinq conceptions [...] obtenues [par précision] [...] peuvent être appelées catégories. Â savoir,

L'être

La qualité (référence à un fondement)

La relation (référence à un corrélat)

La représentation (référence à un interprétant)

La substance

Les trois conceptions intermédiaires peuvent être appelées accidents. (1.555)

Étant donné qu'une catégorie ne peut être préscindée de celle qui est au-dessus d'elle, la liste de leurs objets possibles est la suivante:

Ce qu'est

Le quale (ce qui renvoie à un fondement)

Le relat (ce qui renvoie au fondement et au corrélat)

Le representamen (ce qui renvoie au fondement, au corrélat et à l'interprétant)

Ça. (1.557)

Je voudrais d'abord faire quelques remarques d'ordre général. Le lecteur anglophone est dans une meilleure situation que celle d'un traducteur, quel qu'il soit. Il n'a pas à se soucier du mot à employer. Il lit celui que Peirce lui propose. A lui de se dire que Peirce donnera plus tard dans un autre contexte une signification différente aux mots qu'il emploie dans ce texte. Le traducteur est obligé, lui, de garder les termes, sous peine de commettre un autre contresens, à savoir: faire dire à Peirce en 1867 des choses qu'il ne pouvait pas dire à cette date parce qu'il prônait alors une phénoménologie kantienne fondée sur une logique aristolélicienne, - et non encore la phanéroscopie triadique fondée sur une logique des relations qu'il exposera et défendra après 1885 (pour 
donner une date certifiée par des textes). Il reste au commentateur d'expliquer historiquement le sens des mots et au traducteur de ne traduire que les textes de la période où l'auteur est parvenu à une conception cohérente de son système. C'est ce que j'ai fait dans ma traduction des Écrits sur le signe où ne figurent dans la traduction que des textes postérieurs à 1885 . Je suis ici dans la situation du commentateur. Prenons les passages cités point par point.

Dès 1867 , et même déjà en 1866 , Peirce ne se satisfait plus des catégories aristolélico-kantiennes. Il ne s'agit pas pour lui d'abord de les remplacer par d'autres, mais d'y réfléchir d'une manière critique. Et en premier lieu de poser la question du passage de l'être à la substance. Il y a là un bond inexpliqué. Peirce, qui ne rejette pas encore l'être et la substance, tente de jeter un pont entre l'être et la substance à partir de l'être. La substance est chronologiquement première, mais l'être est logiquement premier. Logiquement, la conception de l'être naît de la formation d'une proposition. Or une proposition comprend un terme pour exprimer une substance ec un terme pour exprimer la qualité de cette substance. Cette conception de la proposition est aristotélicienne, mais elle permet à Peirce de poser comme première étape pour passer de l'être à la substance: la qualité ou référence à un fondement. La deuxième étape à franchir est plus facile à trouver: une qualité est ce qu'elle est parce qu'elle diffère d'autre chose. La relation ou référence à un corrélat constitue donc la deuxième étape. La troisième étape va de soi, car, pour comparer, il faut un point de comparaison, et donc une idée médiatrice ou plus exactement, écrit Peirce,

une représentation médiatrice qui représente le relat comme tenant lieu d'un corrélat avec lequel la représentation médiatrice est elle-même en relation. (1.553)

Deux observations préliminaires:

1) Peirce jette ici la base de ce qui deviendra sa théorie de la hiérarchie des catégories dont nous avons déjà parlé et qui s'obtient par une distinction qu'il appelle «précision» qu'il emprunte à Duns Scot déjà: la praecision de «praescindere» (séparer), qu'il faut distinguer de la "discrimination» et de la «dissociation». La précision introduit un ordre ordinal dans les catégories, comme nous l'avons déjà dit: la représentation (dont l'objet est le representamen) présuppose la relation (dont l'objet est le relat) qui présuppose lui-même la qualité (dont l'objet est le quale).

2) Si ce texte est important de ce point de vue, il est fort gênant à bien d'autres égards, car il introduit la confusion chez ceux qui veulent s'appuyer sur lui pour expliquer les théories 
ultérieures de Peirce. Que ces trois conceptions deviendront les catégories fondamentales de la phanéroscopie est une chose. C'en est une autre que d'utiliser leur définition hors contexte. S'agit-il du même representamen qui deviendra premier dans la théorie ultérieure? La qualité qui est première ici, mais première intermédiaire, est-elle la qualité dont Peirce dira qu'elle est possibilité?

Dans la théorie phanéroscopique achevée, le representamen sera premier et trichotomisable en possible ou qualitatif, en existant et en loi. Si le representamen est premier, peut-on encore le comprendre dans le sens qu'a «représentation» dans le texte de 1867 ? Si la qualité est simplement possible, peut-elle être référence à un fondement? Nous sommes dans un autre monde «pré-peircien", dirions-nous, encore très aristotélico-scolastique, voire thomiste. Peirce n'écrit-il pas dans un article de l'année suivante:

Dans la mesure où la sensation est une simple impression (feeling) d'une sorte particulière, elle n'est déterminée que par une puissance occulte inexplicable; et partant elle n'est pas une représentation [...]. Une impression en tant qu'impression est donc simplement la qualité matérielle d'un signe mental. (5.291)

Qualité matérielle dont le fondement est une «abstraction pure» à laquelle on se réfère, dit Peirce, comme à «une qualité ou un attribut général» (1.551).

C'est à un thomisme revu et corrigé par Kant qu'on a affaire ici: il y a continuité de l'être et du connaître, bien que l'être soit inconnaissable et que le connaître soit un construit transcendantal. L'être et le connaître s'ignorent, mais sont deux aspects du Même. La qualité matérielle de l'être est l'espèce impresse des scolastiques: species coloris est in aere: per aerem ad pupillam defertur; la qualité mentale en est l'espèce expresse (species expressa): ista actione [scilicet visu rei exterioris] visus non videt se, sed colo$\mathrm{rem}^{3}$.

Dans la théorie peircienne constituée, il n'y aura plus de fondement, sauf à l'intérieur même du signe-action, loin de l'être et sans lien avec lui. Ce que Peirce appellera le qualisigne, pur possible dont la possibilité même n'est connue qu'après être venue à l'existence dans un sinsigne, et dont seul un légisigne, à l'intérieur d'un processus sémiotique donné, foumira le sens ou la justification.

3. Cf. Joseph de Tonquédec, la Critique de la connaissance (Paris, Beauchêne, 1929), pp. 55 et 61. 
Il n'y aura plus de substance, mais uniquement des relations, certes productrices d'objets, mais à l'intérieur du système de signes en procès. La proposition ne sera plus constituée d'un terme substantif et d'un terme prédicatif pour exprimer la qualité de cette substance. La proposition est un prédicat ou fonction propositionnelle ou verbe (Peirce l'appelle «rhème») dont l'indice qui la constitue comme proposition donne au verbe l'exister.

La difficulté de lire Peirce vient donc en premier lieu de l'impossibilité de traduire Peirce en ses propres termes sans tenir compte de la date des écrits dans les termes desquels on veut le traduire pour le faire comprendre. La solution est affaire d'érudition.

II. Nous allons garder le même sujet, celui du signe-representamen, pour répondre à la deuxième question que nous posions. A qui Peirce s'adressait-il? Car la difficulté de la traduction historique se complique quand l'auteur essaie de convaincre en employant une terminologie qu'il condamne, pour se faire comprendre. Il faut bien, dit souvent Peirce, jeter des gâteaux à Cerbère pour communiquer. Soit, encore faut-il que le lecteur, le traducteur et le commentateur en tiennent compte pour traduire Peirce.

A s'en tenir au seul mot representamen, pris comme signe formel dans la triade, par opposition au signe-action qui est l'expression qui désigne le processus triadique de la sémiose, on distinguera quatre périodes dans le développement de la pensée de Peirce.

1. Jusqu'en 1873 , Peirce emploie representamen au sens d'objet de la représentation.

2. De 1873 à 1895 , il abandonne le mot.

3. De 1895 à 1903, il le réintroduit.

4. De 1904 à 1911, il l'abandonne de nouveau, sauf dans quatre cas que nous examinerons.

1. De la première période (1866-1873), nous avons amplement discuté. Il ne s'agit pas ici de se faire comprendre. Tout le monde ne se comprend que trop bien. Nous sommes dans un monde dualiste et il faut bien que les mots correspondent aux choses. On accepte le fait. Le comment seul soulève un problème.

2. Labandon du mot «representamen» dans la deuxième période (1873-1895) n'a rien à voir avec la sémiotique phanéroscopique. Peirce s'occupe d'autre chose. Il voyage dans le monde, en Europe surtout, pour le compte du Service Géodésique des États-Unis. La seule innovation - ô combien importante! - de cette période est la suggestion qui deviendra maxime, puis mouvement philosophique, à savoir que ce n'est ni l'autorité ni la raison (forme déguisée de l'autorité) qui décident de la signification d'un 
mot, mais l'action - non l'action individuelle, mais la mise à l'épreuve publique. C'est le pragmatisme.

3. La réintroduction du mot au cours de la troisième période (1895-1903) correspond à l'élaboration logique et phanéroscopique de la sémiotique peircienne, où sont clairement distingués le signe-representamen, définition formelle de chaque élément de la triade et de ses trichotomies formelles, et le signe-action qui est le processus sémiotique proprement dit, qui met en cuvre le representamen, l'interprétant et l'objet (tous trois formellement representamens).

Le representamen n'est plus l'objet d'une représentation. Il $n$ 'est pas non plus un phénomène psychique. Il est là dans le signe-action comme le représentant au sens de "député», "délégué» ou "ambassadeur», de quelque chose d'autre auquel il ne ressemble pas, bien qu'il puisse lui ressembler.

Un signe, ou representamen, est quelque chose qui tient lieu pour quelqu'un de quelque chose sous quelque rapport ou à quelque titre. (2.228, ES: 121)

Peirce limite dès lors

le mot représentation à l'opération d'un signe ou à sa relation à l'objet pour l'interprète de la représentation. Le sujet concret qui représente, [il] l'appelle signe ou representamen. (1.540, ES: 116)

Tout signe est representamen.

Par signe, j'entends tout ce qui communique une notion définie d'un objet de quelque façon que ce soit, étant donné que ces communications de pensée nous sont familières. Partant de cette idée familière, je fais la meilleure analyse que je peux de ce qui est essentiel à un signe et je définis un representamen comme étant tout ce à quoi cette analyse s'applique. (1.540, ES: 116)

D'où la définition formelle du representamen:

UN REPRESENTAMEN est le sujet d'une relation triadique avec un second appelé son OBJET, POUR un troisième appelé son INTERPRÉTANT, cette relation triadique étant telle que le REPRESENTAMEN détermine son interprétant $d$ entretenir la même relation triadique avec le même objet pour quelque interprétant. (1.541, ES: 117)

Mais tout representamen n'est pas un signe. Si un representamen n'a pas d'interprétant mental, il n'est pas un signe.

Ainsi, si une fleur de tournesol, en se tournant vers le soleil, devenait par cet acte même pleinement capable, sans autre condition, de reproduire une fleur de tournesol qui se toume 
vers le soleil exactement de la même façon, et de faire cela avec la même capacité reproductrice, la fleur de tournesol serait un representamen du soleil. (2.274, ES:148)

«Mais», poursuit Peirce, comme à regret, «la pensée est le principal, sinon le seul mode de représentation» (2.274, ES: 148), sous-entendu, que l'homme connaisse.

4. La quatrième période (1903-1911), qui coïncide avec l'explosion et la vogue du pragmatisme, est aussi celle où Peirce trouve une lectrice attentive ou, à tout le moins, bien intentionnée en l'auteur de What is Meaning? à qui Peirce explique son système en des termes qui sont moins les siens que ceux de Lady Welby: le signe, la signification où se rencontrent la sémiotique de Peirce et la signifique de Lady Welby, et les trois divisions de l'interprétant en immédiat, dynamique et final, dont l'intérêt est grand et la fécondité indiscutable, à condition de ne pas réduire la théorie peircienne à un décalque des trois types de signification de Lady Welby: le sens, la signification et la signifiance, et de ne pas oublier que ces interprétants présupposent un interprétant formel dont ils ne sont pas une simple tripartition.

C'est aussi la période où, le succès du pragmatisme aidant, Peirce est sollicité davantage et écrit des articles dans des revues et collabore à des dictionnaires, et où, ici comme là, la discrétion terminologique est recommandée, sinon imposée. Mais aussi bien dans les lettres à Lady Welby que dans les articles de revue et de dictionnaire, le signe - le signe-objet - par opposition au signeaction ou sémiose, est toujours défini comme l'était le representamen. Quelques brèves citations suffiront.

Je définis un Signe comme étant quelque chose qui est si déterminé par quelque chose d'autre, appelé son objet, et qui par conséquent détermine un effet sur une personne, lequel effet $\mathrm{j}$ ' appelle son interprétant, que ce dernier est par là mêtme médiatement déterminé par le premier (ES: 51).

Dans cet extrait d'une lettre à Lady Welby du 23 décembre 1908, Peirce est fort éloigné de la rigueur logique à laquelle il nous a habitués. Faut-il le prendre au pied de la lettre pour comprendre ce qu'est un signe-representamen? Certainement pas, bien que ce soit de cela qu'il s'agit, car Peirce poursuit: «J'ai ajouté «sur une personne», comme pour jeter un gâteau à Cerbère, parce que je désespère de faire comprendre ma propre conception qui est plus large.» (ES: 51) Il aurait pu ajouter: «et plus précise», car l'objet ne détermine pas le signe, comme il le dit, du moins pas l'objet immédiat. Quant à l'objet dynamique, nous ne savons pas ce qu'il est ni comment il exerce son action s'il en exerce une qui ne soit pas purement événementielle. 
Dans sa définition du mot «signe» dans le Dictionnaire de Baldwin, c'est bien le representamen qu'il décrit sous le nom de «signe»:

tout ce qui détermine quelque chose d'autre (son interprétant) à renvoyer à un objet auquel lui-même renvoie (son objet) de la même manière, l'interprétant devenant à son tour un signe et ainsi de suite ad infinitum. (2.303, ES: 126)

Et plus nettement encore dans un manuscrit de 1910, intitulé Meaning:

Le signe ne peut que représenter l'objet et en dire quelque chose. Il ne peut ni faire connaître ni reconnaître l'objet; car c'est ce que veut dire dans le présent volume objet d'un signe; à savoir ce dont la connaissance est présupposée pour pouvoir communiquer des informations supplémentaires le concernant. (2.231, ES: 123)

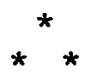

Je terminerai en examinant dans les passages où Peirce utilise, après 1904, le mot «representamen», les raisons formelles qu'il aurait pu avoir pour abandonner ce mot.

Premier passage. Dans un brouillon de lettre à Lady Welby que l'on peut dater par critique interne de juillet 1905 - lettre qu'il n'envoya pas, semble-t-il -, Peirce écrit:

Mon idée en préférant «representamen» [à «signe»] était qu'il semblerait plus naturel de l'appliquer aux députés de nos assemblées, aux délégués de diverses sortes, etc. J'admets toujours que cela aide beaucoup pour comprendre la définition [du signe] de le comparer avec soin à des cas de ce genre. Mais ils s'éloignent certainement de la définition, en ce que celle-ci exige que l'action du signe en tant que telle ne doive pas affecter l'objet représenté. On s'attend, au contraire, qu'un député au parlement, de par sa fonction, améliore la condition de ses mandants, et que n'importe quelle sorte d'avocat affecte la condition de son client. [...] Je concevais le representamen comme prenant la place de la chose, mais un signe n'est pas un substitut. ${ }^{4}$

En bref, de quel signe parlons-nous, du signe-action de la sémiose ou du signe-objet de l'analyse formelle? Celui-ci est bien

4. Semiotics and Significs. The Correspondence between Charles S. Peirce and Victoria Lady Welby. Charles S. Hardwick, ed. (Bloomington, Indiana University Press, 1977), p. 193. 
un representamen: il est là pour la chose, mais il n'en est pas un substitut. Cela n'empêche pas que le député ou le délégué n'agisse en faveur de ses mandants, tout en n'étant pas, par définition, leur substitut. Comme en témoigne dans le même texte cette définition du signe qui n'est autre que la définition formelle du representamen que nous trouvons explicitement de 1895 à 1903 et implicitement de 1904 à 1911.

Un «signe» est n'inporte quoi, A, qui

(1) en plus des autres caractères qui lui sont propres,

(2) entretient une relation dyadique, $R$, avec un corrélat purement actif, $B$,

(3) et également une relation triadique avec B pour un corrélat purement passif, $\mathrm{C}$, cette relation triadique étant telle qu'elle détermine $C$ à être une relation dyadique, $S$, avec $B$, la relation $S$ correspondant incontestablement (in a recognized way) à la relation $R^{5}$

C'est donc plutôt un argument en faveur de l'emploi du mot «representamen» que Peirce nous donne dans ce texte, à condition de ne pas confondre le representamen avec la sémiose ou action du signe.

Deuxième passage. C'est encore de cela qu'il s'agit dans le manuscrit 283 du 8 janvier 1906 où le problème est celui de la correspondance du «signe» avec son «objet». Et Peirce de préférer «representamen» à «signe»: «La Vérité est la conformité d'un representamen à son objet, son objet, je dis bien SON objet.» Mais la correspondance ne suffit pas: «Il doit y avoir une action de l'objet sur le signe pour le rendre vrai. Sans cela, l'objet n'est pas l'objet du representamen.» (5.554)

Ce qui est en question, une fois encore, n'est pas la sémiose, mais le pourquoi de la vérité de la relation du representamen et de l'objet dynamique. Que la sémiose y ait son rôle à jouer, c'est un fait, mais par le moyen de l'objet immédiat que propose l'interprétant.

Troisième passage. Même question encore dans une lettre au Cousin Jo, du 26 juin 1909. L'intérêt de cette lettre est que Peirce y définit le signe comme étant le representamen d'une représentation, les diagrammes mathématiques servant de modèles. Si le raisonnement doit mener quelque part,

il est nécessaire d'avoir une représentation concrète de l'état de choses antérieur (premised), ce representamen étant sus-

5. Ibid., p. 192. 
ceptible de modification, et c'est en expérimentant sur l'effet des modifications que l'on découvre de nouvelles vérités.

Et Peirce de définir le signe comme representamen.

Je définis le signe comme tout ce qui - qu'il soit une chose existante ou un fait réel (actual), ou qu'il soit, comme ce que nous appelons un «mot», une simple forme (form) possible à laquelle un son audible, une configuration (shape) visible, ou un autre objet sensible puisse se conformer, ou qu'il soit une propriété ou une habitude de comportement de quelque chose dont on a fait l'expérience ou que l'on a imaginé - qui est, d'un côté, si déterminé (c'est-à-dire affecté soit par une cause soit par le moyen d'un esprit) par un objet autre que lui-même et, d'un autre côté, affecte à son tour de la même manière un esprit, ou est capable de l'affecter, si bien que cet esprit soit de ce fait médiatement déterminé lui-même par le même objet. $^{6}$

Quatrième passage. Le dernier texte où apparaît le mot «representamen» date de 1911. Il est extrêmement complexe, plein de ratures et de réécritures. Ce qui ressort de tout cela est que la logique est la science, «non de tous les signes ou véhicules d'influence psychique», mais «la science des seuls «Representamens»». Ces derniers mots immédiatement raturés sont remplacés par «une seule sorte de signes auxquels, pour l'instant, je donnerai 1'appellation provisoire de «logons»». Et qu'est-ce qu'un «logon»? Un légisigne ou representamen troisième (Ms. 675). La boucle est bouclée.

Comme le disait le commentateur de Peirce, George A. Benedict, à qui nous devons une analyse minutieuse de ce manuscrit:

[...] les sémioticiens devraient faire usage de ce terme [urepresentamen»] parce que (1) la fonction décrite est toujours sans un terme qui ne soit pas ambigu pour la dénoter, (2) il fournit un remède efficace à l'ambiguité quasi vicieuse dont le mot «signe» est infecté, (3) il rendrait plus difficile à ceux qui travaillent en théorie des signes d'oublier la nature triadique du signe et (4) l'apport de Peirce dans ce domaine est tel que ce serait un honneur mérité que de faire usage du terme «representamen» dont il est l'inventeur et sans lequel la sémiotique ne serait pas ce qu'elle est. ${ }^{7}$

6. Ch. S. Peirce, New Elements of Mathematics, Carolyn Eisele, ed. (La Haye, Mouton, 1976), 3:283.

7. George A. Benedict, "What is a Representamen?» $2^{\circ}$ Congrès de l'Association Internationale de Sémiotique, Vienne, Autriche, 3 juillet 1979. 
Nous voudrions, en guise de conclusion, revenir sur le problème de la traduction en général. Bien entendu, la manière dont nous avons traité la «traduction» de la terminologie de Peirce, tant du point de vue interprétatif que linguistique, mériterait un plus long développement que nous ne pouvons donner ici. Voici en bref ce que nous suggérons. Il y a trois types de traduction au sens classique de transposition d'une langue dans une autre (la question de l'interprétation relevant dans ce cas du traducteur et non du lecteur). 1) On traduit le representamen, autrement dit, on donne dans la langue d'arrivée un décalque du «texte» de la langue de départ. C'est ce qu'a fait pour la traduction de la Bible en français, André Chouraqui, et ce qu'ont fait pour le Talmud les traducteurs de la collection «les Dix paroles» dirigée par Charles Mopsik (Éditions Verdier). Ce travail classique (il fut courant pour le grec et le latin quand on enseignait encore le grec et le latin) requiert un grand nombre de notes ou, de la part du lecteur, une connaissance si parfaite de la langue de départ qu'à la limite la traduction n'a plus de raison d'être. 2) On traduit les objets du discours. Encore faut-il, dans ce cas, que le lecteur connaisse par ailleurs la civilisation des locuteurs de la langue de départ. Cette méthode fut parfois utilisée dans la traduction des textes grecs et latins et en particulier dans des dictionnaires. Elle n'est pas très heureuse. Elle nécessite, elle aussi, un apport extérieur: photos ou dessins et notes explicatives, car les images ne parlent pas. 3) On traduit les interprétants des representamens de la langue de départ. Des ouvriers anglais faisant la pause et buvant une tasse de thé, se traduira en français par des ouvriers avalant un ballon de rouge ou un «blanc sur le zinc». La Collection "Série Noire» de Marcel Duhamel s'est spécialisée, pour le plus grand plaisir des lecteurs, dans ce type de traduction qui n'hésite pas non plus à jouer du décalque des deux méthodes précédentes pour donner un ton d'authenticité au récit, même et surtout dans des romans se passant aux États-Unis et écrits par des auteurs français.

L'idéal serait de conjuguer les trois manières. Et les écrits philosophiques se prêtent assez facilement à ce traitement. C'est dans cet esprit que nous avons traduit Peirce en essayant de ne trahir ni le «texte»-representamen de la langue de départ, ni les concepts-objets exprimés par les representamens, ni les representamens-interprétants de la langue d'arrivée. Encore faut-il connaître la philosophie et son histoire. 